\title{
OBSERVABILITY OF POLYNOMIALLY STABLE SYSTEMS
}

\author{
BIRGIT JACOB AND ROLAND SCHNAUBELT
}

\begin{abstract}
For finite-dimensional systems the Hautus test is a well-known and easy checkable condition for observability. Russell and Weiss (SIAM J. Control Optim. 32:1$23,1994)$ suggested an infinite-dimensional version of the Hautus test, which is necessary for exact infinite-time observability and sufficient for approximate infinite-time observability of exponentially stable systems. In this paper the notion of observability is studied for polynomially stable systems. Several known results for exponentially stable systems are extended to the setting of polynomially stable systems. By means of an example the obtained results are illustrated.
\end{abstract}

\section{INTRODUCTION}

In this paper we study the observability of the system

$$
\begin{aligned}
\dot{x}(t) & =A x(t), & t \geq 0, \\
y(t) & =C x(t), & t \geq 0, \\
x(0) & =x_{0}, &
\end{aligned}
$$

on a Banach space $X$. We assume that $A$ is the infinitesimal generator of a strongly continuous semigroup $T(\cdot)=(T(t))_{t \geq 0}$ on $X$, and that $C$ is a linear bounded operator from the domain of $A$, denoted by $X_{1}:=D(A)$, to another Banach space $Y$. Here we have equipped $D(A)$ with the graph norm. By a solution of $\dot{x}(t)=A x(t)$ with initial condition $x(0)=x_{0} \in X$ we mean the continuous function

$$
x(t)=T(t) x_{0}, \quad t \geq 0 .
$$

These assumptions are not sufficient to guarantee that the output of the system, the function $y(\cdot)$, is an element of $L_{\text {loc }}^{2}\left(\mathbb{R}_{+}, Y\right)$. In order to guarantee this, we assume that $C$ is an admissible observation operator for $T(\cdot)$. The notion of admissible observation operators was introduced by Weiss [22] as follows.

Definition 1.1. An operator $C \in \mathcal{B}\left(X_{1}, Y\right)$ is an admissible observation operator for $T(\cdot)$ if the map $\Psi_{\infty} x=C T(\cdot) x$ (initially defined on $X_{1}$ ) has a continuous extension $\Psi_{\infty}: X \rightarrow L_{\text {loc }}^{2}\left(\mathbb{R}_{+}, Y\right)$ (where $L_{\text {loc }}^{2}$ is endowed with the usual Fréchet topology).

Here $\mathcal{B}(X, Y)$ denotes the set of bounded linear operators from $X$ to $Y$. For further information concerning admissibility we refer the reader to the survey [10]. In this paper we discuss in particular the following observability concepts.

2000 Mathematics Subject Classification. Primary: 93B07. Secondary: 47D06, 93C25.

Key words and phrases. Polynomially stable systems, approximate observability, exact observability, admissibility, Hautus test. 
Definition 1.2. The system (1.1) is called approximately infinite-time observable if $\Psi_{\infty}$ : $X \rightarrow L_{\text {loc }}^{2}\left(\mathbb{R}_{+}, Y\right)$ is injective. If

$$
\left\|\Psi_{\infty} x\right\|_{L^{2}\left(\mathbb{R}_{+}, Y\right)} \geq \kappa\|x\|
$$

holds for a constant $\kappa>0$ and for every $x \in X$, then the system (1.1) is called exactly infinite-time observable. (Here the left hand side is taken to be $\infty$ if $\Psi_{\infty} x \notin L^{2}\left(\mathbb{R}_{+}, Y\right)$.)

It is well known that for reflexive Banach spaces $X$ and $Y$ the concept of admissible observation operators is dual to that of admissible control operators (see e.g. Salamon $[21]$ ), and the notion of approximate (exact) infinite-time observability is dual to approximate (exact) infinite-time controllability (see e.g. Dolecki and Russell [6]). Controllability and observability are important properties of a distributed parameter system, which have been extensively studied in the literature, see for example [2], [14] and [19].

The Hautus Lemma, due to Popov [18] and Hautus [9], is a powerful and well known test for observability of finite-dimensional systems. It states that the system (1.1) with $A \in \mathbb{C}^{n \times n}$ and $C \in \mathbb{C}^{p \times n}$ is observable if and only if

$$
\operatorname{rank}\left[\begin{array}{c}
s I-A \\
C
\end{array}\right]=n \quad \text { for all } s \in \mathbb{C} .
$$

Russell and Weiss [20] proposed the following generalization of the Hautus test to the infinite-dimensional situation: There exists a $m>0$ such that

$$
\|(s I-A) x\|^{2}+|\operatorname{Re}(s)|\|C x\|^{2} \geq m|\operatorname{Re}(s)|^{2}\|x\|^{2}
$$

for all complex $s$ with negative real part and for all $x \in D(A)$. Under the assumption that the semigroup $T(\cdot)$ is exponentially stable, they showed that condition (RW) is necessary for the exact infinite-time observability of (1.1) and that it is sufficient for the approximate infinite-time observability of (1.1). In several situations, such as if $A$ is bounded [20], $A$ is a Riesz-spectral operator and $\operatorname{dim} Y<\infty$ [11], or $A+\omega I$ is skew-adjoint [16], [23], the Hautus test is in fact sufficient for exact infinite-time observability. However, in general the Hautus test does not imply exact infinite-time observability even for exponentially stable semigroups, [12]. A counterexample in [13] shows that for strongly stable systems, that is, $T(t) x \rightarrow 0$ as $t \rightarrow \infty$ for all $x \in X$, the Hautus test is not a sufficient condition for approximate infinite-time observability.

Certain weakly damped or coupled wave equations lead to semigroups $T(\cdot)$ which do not decay exponentially, but it holds

$$
\|T(t) x\| \leq c t^{-\beta}(\|x\|+\|A x\|) \quad \forall t \geq 0, x \in D(A)
$$

for some constants $c, \beta>0$; see [1], [3], and the references therein. In these situations the spectrum of $A$ typically belongs to the open left halfplane and approaches $i \mathbb{R}$ at $\pm i \infty$. In Section 2 we briefly recall the relevant definitions and properties of such polynomially stable semigroups. We note that every exponentially stable semigroup is polynomially stable, and that every bounded, polynomially stable semigroup is strongly stable.

The above indicated results concerning the Hautus test cannot be applied to polynomially stable semigroups. (One could apply them to the rescaled exponentially stable semigroup $\left(e^{-\omega t} T(t)\right)_{t \geq 0}$ with $\omega>0$, but this would give rather crude results; e.g., the behavior of the spectrum of $A$ near $i \mathbb{R}$ would be ignored.) In this paper we want to study 
the Hautus test for polynomially stable semigroups. For these investigations it turns out to be useful to introduce variants of the Hautus test (RW) and of approximate and exact infinite time observability where one replaces $X$ by fractional power spaces $D\left((w I-A)^{\alpha}\right)$. These concepts are defined and discussed in Section 3. In particular, in Definition 3.5 we introduce the $(\alpha, 0)$-Hautus test which is weaker than the Hautus test (RW). The main result of this paper is as follows.

Theorem 1.3. Assume that the semigroup $T(\cdot)$ is polynomially stable. Then the $(\alpha, 0)$ Hautus test is sufficient for the approximate infinite-time observability of the system (1.1) and necessary for the exact infinite-time observability of the system (1.1).

Theorem 1.3 is a special case of Proposition 3.6 (for the necessity part) and of Theorem 3.10 (for the sufficiency part). We point out that Theorem 3.10 improves Theorem 1.6 of [20] even in the case exponentially stable semigroups. As in the case of exponentially stable semigroups, the theory can be complemented by several results concerning infinite time admissibility, finite time exact observability and sufficient conditions for exact infinite-time observability, see Section 3. We conclude the paper with an extended example illustrating our concepts and results in the case of diagonal systems. This example also shows that the exponents $\alpha$ in our results are optimal in several respects.

\section{Preliminaries on polynomially Stable SEMigroups}

In this section we fix our notation and review some results on polynomially stable $C_{0}$-semigroups. By $D(B), N(B) \sigma(B), \rho(B)$, we denote the domain, kernel, spectrum, resolvent set of a linear operator $B$, respectively, and we set $R(\lambda, B)=(\lambda I-B)^{-1}$. The open left and right half planes of $\mathbb{C}$ are designated by $\mathbb{C}_{-}$and $\mathbb{C}_{+}$, respectively. We write $c=c(\alpha, \beta, \cdots)$ for a generic constant depending on the quantities $\alpha, \beta, \cdots$. Throughout this paper, $A$ is the generator of a $C_{0}$-semigroup $T(\cdot)$ on a Banach space $X$.

Fix a real number $w$ such that $\|T(t)\| \leq M e^{(w-\varepsilon) t}$ for some constants $M, \varepsilon>0$ and all $t \geq 0$. We define the fractional powers of $A_{w}:=w I-A$ by

$$
A_{w}^{-\alpha}=\frac{1}{2 \pi i} \int_{\Gamma}(w-\lambda)^{-\alpha} R(\lambda, A) d \lambda
$$

where $\alpha>0$ and $\Gamma$ is any piecewise smooth path in the set $\{\lambda \in \mathbb{C}: \operatorname{Re} \lambda>w-\varepsilon, \lambda \notin$ $[w, \infty)\}$ running from $\infty e^{-i \phi}$ to $\infty e^{i \phi}$ for some $0<\phi<\pi / 2$, see [7, Section II.5] or [17, Section 2.7]. We further set $A_{w}^{0}=I$. The operator $A_{w}^{-\alpha}$ is injective and bounded, hence it has a closed inverse denoted by $A_{w}^{\alpha}$. We endow the domain $D\left(A_{w}^{\alpha}\right)=: X_{\alpha}$ with the norm $\left\|A_{w}^{\alpha} x\right\|_{X}=:\|x\|_{\alpha}, \alpha \geq 0$, where $X_{0}=X$. Observe that $X_{\beta}$ is continuously and densely embedded in $X_{\alpha}$ for $\beta \geq \alpha \geq 0$ and that $\|x\|_{n}$ is equivalent to the usual graph norm of $A^{n}$ for $n \in \mathbb{N}$. Moreover, the fractional powers commute with $T(t)$ and $A$.

Definition 2.1. A $C_{0}$-semigroup $T(\cdot)$ is called polynomially stable if there are constants $\alpha, \beta>0$ such that

$$
\left\|T(t) A_{w}^{-\alpha}\right\| \leq N t^{-\beta}
$$

for a constant $N>0$ and all $t \geq 1$.

We note that inequality $(2.1)$ is equivalent to

$$
\|T(t) x\| \leq N t^{-\beta}\|x\|_{\alpha} \quad \forall x \in X_{\alpha} .
$$


Observe that estimate (2.1) with $\alpha=0$ and $\beta>0$ already implies that $T(\cdot)$ is exponentially stable, i.e. $\|T(t)\| \leq M e^{-\varepsilon t}$ for $t \geq 0$ and some constants $M, \varepsilon>0$. It was shown in [3, Proposition 3.1] that a polynomially stable semigroup satisfies

$$
\left\|T(t) A_{w}^{-\alpha \gamma}\right\| \leq c(\gamma) t^{-\beta \gamma}, \quad t \geq 1,
$$

for each $\gamma \geq 1$. Moreover, inequality (2.3) holds for all $\gamma>0$ if $T(\cdot)$ is polynomially stable and bounded (i.e., $\|T(t)\| \leq M$ for some constant $M \geq 1$ and all $t \geq 0$ ). For every semigroup $T(\cdot)$, estimate $(2.1)$ with $\beta=1$ and $\alpha>0$ implies that $\sigma(A) \subset \mathbb{C}_{-}$and that

$$
\left\|R(\lambda, A) A_{w}^{-\alpha-\varepsilon}\right\| \leq c(\varepsilon), \quad \operatorname{Re} \lambda \geq 0,
$$

for each $\varepsilon>0$, see [3, Proposition 3.3]. Conversely, if $T(\cdot)$ is bounded and $\left\|R(\lambda, A) A_{w}^{-\alpha}\right\|$ is bounded for $\lambda \in i \mathbb{R}$, then

$$
\left\|T(t) A_{w}^{-\alpha-\varepsilon}\right\| \leq c(\varepsilon) t^{-1}, \quad t \geq 1,
$$

for each $\varepsilon>0$ due to [3, Theorem 3.5]. We further remark that

$$
\left\|R(\lambda, A) A_{w}^{-\alpha}\right\| \leq c \quad \forall \operatorname{Re} \lambda \geq 0 \quad \Longleftrightarrow \quad\|R(\lambda, A)\| \leq c\left(1+|\lambda|^{\alpha}\right) \quad \forall \operatorname{Re} \lambda \geq 0
$$

for each generator $A$ with $\sigma(A) \subset \mathbb{C}_{-}$, see e.g. [3, Proposition 3.6]. There are bounded, polynomially stable semigroups arising from coupled wave equations whose generator spectrum $\sigma(A)$ is contained in $\mathbb{C}_{-}$and approaches $i \mathbb{R}$ at $\pm i \infty$ (so that these semigroups are not exponentially stable). It may also happen that $T(\cdot)$ is polynomially stable, but $\|T(t)\|$ grows exponentially as $t \rightarrow \infty$ and $\sigma(A) \subset\{\lambda \in \mathbb{C}: \operatorname{Re} \lambda \leq-1\}$. We refer to [1] and [3] for such examples and further results and references.

\section{OBSERVABILITy CONCEPtS OF POLYNOMIALly STABlE SYSTEMS}

We now return to infinite-dimensional systems described by (1.1). Throughout this section we assume that $A$ generates a $C_{0}$-semigroup $T(\cdot)$ on a Banach space $X$ and that $C$ is an admissible observation operator for $T(\cdot)$.

It is known that if $T(\cdot)$ is exponentially stable, then $C$ is infinite-time admissible; i.e., $\Psi_{\infty}: X \rightarrow L^{2}\left(\mathbb{R}_{+}, Y\right)$ is bounded. In the following lemma we prove a similar fact for polynomially stable semigroups. Observe that we can always assume that $\beta>1 / 2$ in (2.1) due to (2.3), possibly after increasing the initially given $\alpha$.

Lemma 3.1. Assume that $T(\cdot)$ is polynomially stable with constants $(\alpha, \beta)$, where $\beta>$ $1 / 2$. Then $\Psi_{\infty}: X_{\alpha} \rightarrow L^{2}\left(\mathbb{R}_{+}, Y\right)$ is bounded.

Proof. The admissibility of $C$ and inequality (2.2) imply that

$$
\begin{aligned}
\left\|\Psi_{\infty} x\right\|_{L^{2}\left(\mathbb{R}_{+}, Y\right)}^{2} & =\sum_{k=0}^{\infty} \int_{0}^{1}\|C T(t) T(k) x\|^{2} d t \leq c \sum_{k=0}^{\infty}\|T(k) x\|^{2} \\
& \leq c \sum_{k=0}^{\infty} k^{-2 \beta}\|x\|_{\alpha}^{2}=c(\beta)\|x\|_{\alpha}^{2}
\end{aligned}
$$

for $x \in X_{1+\alpha}$. This estimate yields the assertion.

We see in Section 4 that the value of $\alpha$ in the above result is almost sharp. Next we generalize the notion of exact infinite-time observability. 
Definition 3.2. Let $\alpha \geq 0$. The system (1.1) is $\alpha$-exactly infinite-time observable if

$$
\left\|\Psi_{\infty} x\right\|_{L^{2}\left(\mathbb{R}_{+}, Y\right)} \geq \kappa\|x\|_{\alpha}
$$

holds for some constant $\kappa>0$ and for every $x \in X_{\alpha}$. (Here the left hand side is taken to be $\infty$ if $\Psi_{\infty} x \notin L^{2}\left(\mathbb{R}_{+}, Y\right)$.)

Observe that $\alpha$-exact infinite-time observability implies $\beta$-exact infinite-time observability if $\alpha \geq \beta \geq 0$. An $\alpha$-exactly infinite-time observable system may loose this property if one increases the exponent $\alpha$, see Section 4. Obviously, 0-exact infinite-time observability coincides with exact infinite-time observability.

We further note that the system (1.1) is $\alpha$-exactly infinite-time observable if and only if there is a constant $\kappa^{\prime}>0$ such that for each $x \in X_{\alpha}$ there is a constant $t_{x}>0$ with

$$
\left\|\Psi_{\infty} x\right\|_{L^{2}\left(\left(0, t_{x}\right), Y\right)} \geq \kappa^{\prime}\|x\|_{\alpha} .
$$

If $\alpha=0$ and $T(\cdot)$ is exponentially stable, then $t_{x}$ can be chosen independently of $x \in X$ by [20, Proposition 2.8]. In other words, if $T(\cdot)$ is exponentially stable, then an exactly infinite-time observable system is in fact exactly observable in a finite time $t_{0}>0$. We obtain similar results for polynomially stable systems.

Proposition 3.3. Assume that $T(\cdot)$ is polynomially stable and bounded. Let $C$ be an infinite-time admissible observation operator for $T(\cdot)$ and let $\alpha>0$. Then the following statements are equivalent.

(a) The system (1.1) is $\alpha$-exactly infinite-time observable.

(b) There exist constants $\kappa^{\prime}, t_{0}>0$ such that

$$
\left\|\Psi_{\infty} x\right\|_{L^{2}\left(\left(0, t_{0}\right), Y\right)} \geq \kappa^{\prime}\|x\|_{\alpha} \quad \forall x \in X_{\alpha} .
$$

Proof. The implication ' $(\mathrm{b}) \Rightarrow(\mathrm{a})$ ' is obvious. Let the system (1.1) be $\alpha$-exactly infinitetime observable. Using this assumption, the infinite-time admissibility and (2.2), we estimate

$$
\begin{aligned}
\kappa\|x\|_{\alpha} & \leq\left\|\Psi_{\infty} x\right\|_{L^{2}\left(\mathbb{R}_{+}, Y\right)} \leq\left\|\Psi_{\infty} x\right\|_{L^{2}\left(\left(0, t_{0}\right), Y\right)}+\left\|\Psi_{\infty} T\left(t_{0}\right) x\right\|_{L^{2}\left(\mathbb{R}_{+}, Y\right)} \\
& \leq\left\|\Psi_{\infty} x\right\|_{L^{2}\left(\left(0, t_{0}\right), Y\right)}+c\left\|T\left(t_{0}\right) x\right\| \leq\left\|\Psi_{\infty} x\right\|_{L^{2}\left(\left(0, t_{0}\right), Y\right)}+c t_{0}^{-\beta}\|x\|_{\alpha},
\end{aligned}
$$

for some constants $\beta, \kappa, c>0$ and every $t_{0}>0$. Taking a sufficiently large $t_{0}>0$, we arrive at assertion (b).

We can weaken the assumptions on $T(\cdot)$ and $C$ in the above proposition if we restrict ourselves to sufficiently large $\alpha$.

Proposition 3.4. Assume that $T(\cdot)$ is polynomially stable with constants $(\bar{\alpha}, \beta)$, where $\beta>1 / 2$. Let $C$ be an admissible observation operator for $T(\cdot)$ and let $\alpha \geq \alpha_{0}:=2 \bar{\alpha}$. Then the following statements are equivalent.

(a) The system (1.1) is a-exactly infinite-time observable.

(b) There exist constants $\kappa^{\prime}, t_{0}>0$ such that

$$
\left\|\Psi_{\infty} x\right\|_{L^{2}\left(\left(0, t_{0}\right), Y\right)} \geq \kappa^{\prime}\|x\|_{\alpha} \quad \forall x \in X_{\alpha} .
$$


Proof. Assume that (a) holds. Then Lemma 3.1 and estimate (2.1) yield

$$
\begin{aligned}
\kappa\|x\|_{\alpha} & \leq\left\|\Psi_{\infty} x\right\|_{L^{2}\left(\mathbb{R}_{+}, Y\right)} \leq\left\|\Psi_{\infty} x\right\|_{L^{2}\left(\left(0, t_{0}\right), Y\right)}+\left\|\Psi_{\infty} T\left(t_{0}\right) x\right\|_{L^{2}\left(\mathbb{R}_{+}, Y\right)} \\
& \leq\left\|\Psi_{\infty} x\right\|_{L^{2}\left(\left(0, t_{0}\right), Y\right)}+c\left\|(\omega I-A)^{\bar{\alpha}} T\left(t_{0}\right) x\right\| \\
& \leq\left\|\Psi_{\infty} x\right\|_{L^{2}\left(\left(0, t_{0}\right), Y\right)}+c t_{0}^{-\beta}\left\|(\omega I-A)^{\bar{\alpha}} x\right\|_{\bar{\alpha}} \\
& \leq\left\|\Psi_{\infty} x\right\|_{L^{2}\left(\left(0, t_{0}\right), Y\right)}+c t_{0}^{-\beta}\|x\|_{\alpha}
\end{aligned}
$$

for some constants $\kappa, c>0$ and every $t_{0}>0$, where we also used $\alpha \geq 2 \bar{\alpha}$. As in the previous proof, assertion (b) follows. The other implication is again obvious.

In order to characterize observable polynomially stable systems, we introduce the following version of the Hautus-test (RW) from the introduction.

Definition 3.5. Let $\alpha, \beta \geq 0$. We say that the system (1.1) satisfy the ( $\alpha, \beta)$-Hautus test if there is a constant $m>0$ such that for all $x \in X_{1+\alpha} \cap X_{\beta}$ and $\lambda \in \mathbb{C}_{-}$there is a constant $m_{\lambda} \geq 0$ with

$$
\|(\lambda I-A) x\|_{\alpha}^{2}+m_{\lambda}\|C x\|^{2} \geq m^{2}|\operatorname{Re} \lambda|^{2}\|x\|_{\beta}^{2} .
$$

If $\alpha=\beta=0$, then we say that (1.1) satisfies the Hautus test.

Observe that the $(\alpha, \beta)$-Hautus test implies the $\left(\alpha^{\prime}, \beta\right)$-Hautus test if $\alpha^{\prime} \geq \alpha \geq 0$ and the $\left(\alpha, \beta^{\prime}\right)$-Hautus test if $0 \leq \beta^{\prime} \leq \beta$. It may happen that a valid Hautus test fails if one decreases $\alpha$ for fixed $\beta$, or if increases $\beta$ for fixed $\alpha$, cf. Section 4 . We remark that on the right hand side of (3.1) the dependence on $\lambda$ can be weakened considerably, see Remark 3.13, but for simplicity we work with the concept given in Definition 3.5.

In view of the Hautus test introduced by Russell and Weiss in [20], see the introduction, we say that the system (1.1) satisfies the $(\alpha, \beta)$-Hautus test (RW) if the system (1.1) satisfies the $(\alpha, \beta)$-Hautus test with $m_{\lambda}=|\operatorname{Re} \lambda|$. The Hautus test (RW) with $\alpha=\beta=0$ is well studied in the literature, as discussed in the introduction. We recall that Russell and Weiss proved in [20] that exact infinite-time observability implies the Hautus test (RW) and that the Hautus-test (RW) implies approximate infinite-time observability, for exponentially stable semigroups. We shall extend several known results for exponentially stable systems to the setting of polynomially stable semigroups, starting with a necessary condition for $\beta$-exact infinite-time observability. In Section 4 we show that this condition cannot be improved, in general.

Proposition 3.6. Let $\alpha, \beta \geq 0$. Suppose that $\Psi_{\infty}: X_{\alpha} \rightarrow L^{2}\left(\mathbb{R}_{+}, Y\right)$ is bounded (e.g., if $T(\cdot)$ is polynomially stable with constants $(\alpha, \bar{\beta})$, where $\bar{\beta}>1 / 2)$ and that the system $(1.1)$ is $\beta$-exactly infinite-time observable. Then the system (1.1) satisfies the $(\alpha, \beta)$-Hautus test (RW).

Proof. We proceed as in [20] where the case $\alpha=\beta=0$ was considered. Let $x \in X_{\alpha+2}$ and $\lambda \in \mathbb{C}_{-}$. Set $e_{\lambda}(t)=e^{\lambda t}$ for $t \geq 0$ and $e_{\lambda}(t)=0$ for $t<0$. We then have

$$
\begin{aligned}
T(t) x & =e^{\lambda t} x+\int_{0}^{t} e^{\lambda(t-s)} T(s)(A-\lambda I) x d s, \quad t \geq 0, \\
\Psi_{\infty} x & =e_{\lambda} C x+e_{\lambda} * \Psi_{\infty}(A-\lambda I) x .
\end{aligned}
$$


Thus Young's inequality and our assumptions imply that

$$
\kappa\|x\|_{\beta} \leq\left\|\Psi_{\infty} x\right\|_{L^{2}\left(\mathbb{R}_{+}, Y\right)} \leq \frac{1}{\sqrt{2|\operatorname{Re} \lambda|}}\|C x\|+\frac{c}{|\operatorname{Re} \lambda|}\|(\lambda I-A) x\|_{\alpha} .
$$

This estimate easily yields the assertion.

Remark 3.7. The proof of Proposition 3.6 also implies the following result: Suppose that $\Psi_{\infty}: X_{\alpha} \rightarrow L^{2}\left(\mathbb{R}_{+}, Y\right)$ is bounded for some $\alpha \geq 0$ and that the system (1.1) is approximately infinite-time observable. Then

$$
\|(\lambda I-A) x\|_{\alpha}^{2}+|\operatorname{Re} \lambda|\|C x\|^{2}>0
$$

for every $x \in X_{1+\alpha} \backslash\{0\}$ and $\lambda \in \mathbb{C}_{-}$.

In the following two propositions we show that for certain classes of systems the $(\alpha, \alpha)$ Hautus test (RW) is even sufficient for $\alpha$-exact infinite-time observability. Recall that a Riesz basis of a Hilbert space $H$ is a sequence $\left(\phi_{n}\right)_{n \in \mathbb{N}}$ in $H$ such that $\phi_{n}=S e_{n}$ for an invertible operator $S \in \mathcal{B}(H)$ and an orthonormal basis $\left(e_{n}\right)_{n \in \mathbb{N}}$ of $H$. A Riesz-spectral operator $A$ on $H$ is an operator possessing a Riesz basis of eigenvectors. We also note that in the following result one can weaken the assumption that $C$ is admissible to $\alpha+1$ admissibility; i.e, $C$ is admissible for the restriction of $T(\cdot)$ to $X_{\alpha}$, see [15].

Proposition 3.8. Let $X$ be a Hilbert space and $A$ be a Riesz-spectral operator. Suppose that the eigenvalues of $A$ are contained in the open left half plane, that $\operatorname{dim} Y<\infty$, and that $\Psi_{\infty}: X_{\alpha} \rightarrow L^{2}\left(\mathbb{R}_{+}, Y\right)$ is bounded for some $\alpha \geq 0$. Then the following statements are equivalent.

(a) The system (1.1) is $\alpha$-exactly infinite-time observable.

(b) The system (1.1) satisfies the $(\alpha, \alpha)$-Hautus test (RW).

Proof. The assertion is a consequence of Theorem 2 in [11] if we consider the system (1.1) on the space $X_{\alpha}$.

The next result improves [8, Theorem 3.2] where it was shown that the Hautus-test (RW) with $m=1$ implies exact infinite-time observability if the semigroup $T(\cdot)$ is exponentially stable. Recall that $T(\cdot)$ is called strongly stable if $T(t) x \rightarrow 0$ as $t \rightarrow \infty$ for all $x \in X$.

Proposition 3.9. Assume that $X$ is a Hilbert space, that $T(\cdot)$ is strongly stable and that $\alpha \geq 0$. If there exists a sequence $\left(s_{n}\right)_{n \in \mathbb{N}} \subset(-\infty, 0)$ such that $\lim _{n \rightarrow \infty} s_{n}=-\infty$ and

$$
\left\|\left(s_{n} I-A\right) x\right\|_{\alpha}^{2}+\left|s_{n}\right|\|C x\|^{2} \geq\left|s_{n}\right|^{2}\|x\|_{\alpha}^{2} \quad \forall n \in \mathbb{N}, x \in X_{1+\alpha},
$$

then the system (1.1) is $\alpha$-exactly infinite-time observable.

Proof. Property (3.2) is equivalent to

$$
\|C x\|^{2}+\frac{1}{\left|s_{n}\right|}\|A x\|_{\alpha}^{2} \geq-\langle A x, x\rangle_{\alpha}-\langle x, A x\rangle_{\alpha} \quad \text { forall } n \in \mathbb{N}, x \in X_{1+\alpha} .
$$

Letting $n \rightarrow \infty$, we obtain for each $t \geq 0$ and $x \in X_{1+\alpha}$ the inequality

$$
\|C T(t) x\|^{2} \geq-\langle A T(t) x, T(t) x\rangle_{\alpha}-\langle T(t) x, A T(t) x\rangle_{\alpha} .
$$


Let $t_{0}>0$. Integrating this inequality from 0 to $t_{0}$, we deduce

$$
\int_{0}^{t_{0}}\|C T(t) x\|^{2} d t \geq\|x\|_{\alpha}^{2}-\left\|T\left(t_{0}\right) x\right\|_{\alpha}^{2}
$$

for $x \in X_{1+\alpha}$. The assertion now follows from this estimate combined with the strong stability of the semigroup $T(\cdot)$.

We now give a sufficient condition for approximate infinite-time observability improving Theorem 1.6 of [20] in two ways: We only require that $T(\cdot)$ is polynomially stable (instead of exponentially stable), and we use the $(\alpha, 0)$-Hautus test instead of the stronger Hautus test (RW). A counterexample in [13] shows that one cannot further reduce polynomial stability to mere strong stability. In the next section we present an approximately infinitetime observable and polynomially stable system which violates the $(\alpha, 0)$-Hautus test for every choice of $\alpha \geq 0$ and $m_{\lambda}>0$; i.e., the converse of Theorem 3.10 does not hold.

Theorem 3.10. Let $T(\cdot)$ be polynomially stable and assume that the system (1.1) satisfies the $(\alpha, 0)$-Hautus test for some $\alpha \geq 0$. Then the system (1.1) is approximately infinitetime observable.

For the proof of Theorem 3.10 we need the following lemma, which relies on the Phragmen Lindelöf principle: Let $Z$ be a Banach space and $f:\{\lambda \in \mathbb{C}: \operatorname{Re} \lambda \geq a\} \rightarrow Z$ be a continuous function which is holomorphic for $\operatorname{Re} \lambda>a$ and satisfies $|f(\lambda)| \leq c e^{b|\lambda|^{\gamma}}$ for $\operatorname{Re} \lambda \geq a$ and constants $a \in \mathbb{R}, c, b \geq 0$, and $0 \leq \gamma<1$. Then it holds

$$
\sup _{\operatorname{Re} \lambda \geq a}\|f(\lambda)\|=\sup _{\operatorname{Re} \lambda=a}\|f(\lambda)\| .
$$

This fact is shown in e.g. [4, Corollary VI.4.2] for $Z=\mathbb{C}$ and can be extended to general $Z$ using linear forms.

Lemma 3.11. Assume that $S(\cdot)$ is a polynomially stable $C_{0}$-semigroup with generator $B$ on a Banach space $Z$ such that

$$
\left\|(\lambda I-B)(w I-B)^{\alpha} x\right\| \geq m|\operatorname{Re} \lambda|\|x\|
$$

for $x \in Z_{1+\alpha}=D\left(B_{w}^{1+\alpha}\right), \lambda \in \mathbb{C}_{-}$, some $\alpha \geq 0$, a sufficiently large $w \in \mathbb{R}$, and a constant $m>0$. Then $Z=\{0\}$.

Proof. Since $S(\cdot)$ is polynomially stable there are constants $\alpha, \beta, N>0$ such that $(2.1)$ holds for all $t \geq 1$. Here we may assume that $\beta=1$. (Use (2.3) with $\gamma>1$ if $\beta \in(0,1)$ initially.) Due to (2.4) and (2.5), we know that $\sigma(B) \subset \mathbb{C}_{-}$and

$$
\|R(\lambda, B)\| \leq K\left(1+|\lambda|^{\gamma}\right)=: \varphi(|\lambda|)
$$

for $\operatorname{Re} \lambda \geq 0$ and some constants $K, \gamma \geq 0$. If $\sigma(B) \neq \emptyset$, then there would exist $\lambda \in \sigma(B)$ and $\lambda_{n} \in \rho(B)$ such that $\operatorname{Re} \lambda_{n} \leq \operatorname{Re} \lambda / 2=:-\delta<0$ for $n \in \mathbb{N}$ and $\lambda_{n} \rightarrow \lambda$ as $n \rightarrow \infty$. Hence, $\left\|R\left(\lambda_{n}, B\right)\right\| \rightarrow \infty$ as $n \rightarrow \infty$. Take a natural number $k \geq \alpha$. The identity

$$
R\left(\lambda_{n}, B\right) B^{-k}=\lambda_{n}^{-k} R\left(\lambda_{n}, B\right)+\sum_{j=1}^{k} \lambda_{n}^{-j} B^{-k-1+j}
$$


yields $\left\|R\left(\lambda_{n}, B\right) B^{-k}\right\| \rightarrow \infty$ as $n \rightarrow \infty$. On the other hand, (3.3) implies that

$$
\left\|R\left(\lambda_{n}, B\right) B^{-k}\right\|=\left\|B_{w}^{\alpha} B^{-k} R\left(\lambda_{n}, B\right) B_{w}^{-\alpha}\right\| \leq \frac{c}{m \delta},
$$

which is a contradiction. As a result, $B$ has empty spectrum.

Let $\lambda \in \mathbb{C}$ and $z \in Z_{\alpha}$. If $0 \geq \operatorname{Re} \lambda \geq-(2 \varphi(|\operatorname{Im} \lambda|))^{-1}$, then

$$
\|R(\lambda, B)\| \leq 2 \varphi(|\operatorname{Im} \lambda|) \leq 2 \varphi(|\lambda|)
$$

by a standard perturbation argument. The estimate (3.3) further yields

$$
\|R(\lambda, B) z\| \leq \frac{1}{m|\operatorname{Re} \lambda|}\|z\|_{\alpha} \leq \frac{2 \varphi(|\operatorname{Im} \lambda|)}{m}\|z\|_{\alpha} \leq \frac{2 \varphi(|\lambda|)}{m}\|z\|_{\alpha}
$$

if $\operatorname{Re} \lambda \leq-(2 \varphi(|\operatorname{Im} \lambda|))^{-1}$. Summing up, we have established that

$$
\|R(\lambda, B) z\| \leq K^{\prime}\left(1+|\lambda|^{\gamma}\right)\|z\|_{\alpha}
$$

on every right half plane $\{\lambda \in \mathbb{C}: \operatorname{Re} \lambda \geq-r\}$. Then [4, Corollary VI.4.2] and (3.3) show

$$
\|R(\lambda, B) z\| \leq \sup _{s \in \mathbb{R}}\|R(-r-i s, B) z\| \leq \frac{\|z\|_{\alpha}}{m r}
$$

for all $\operatorname{Re} \lambda \geq-r$ and $r>0$. Letting $r \rightarrow \infty$, we obtain $R(\lambda, B) z=0$ for all (fixed) $\lambda \in \mathbb{C}$ and $z \in Z_{\alpha}$. The density of $Z_{\alpha}$ in $Z$ implies that $R(\lambda, B)=0$, and thus $Z=\{0\}$.

Proof of Theorem 3.10: We have to show that $Z=N\left(\Psi_{\infty}\right)$ is trivial. The admissibility of $C$ implies that $Z$ is a closed subspace of $X$, and it is easy to see that $Z$ is $T(\cdot)$-invariant. Thus the restriction $\tilde{T}(t)$ of $T(t)$ to $Z$ yields a $C_{0}$-semigroup on $Z$ generated by the restriction $\tilde{A}$ of $A$ to $Z$. Observe that $\tilde{T}(\cdot)$ is still polynomially stable. If $x \in D(\tilde{A})$, then $C x=\left(\Psi_{\infty} x\right)(0)=0$. Thus the $(\alpha, 0)$-Hautus test implies that

$$
\left\|(\lambda I-\tilde{A})(w I-\tilde{A})^{\alpha} x\right\| \geq m|\operatorname{Re} \lambda|\|x\|
$$

for $\lambda \in \mathbb{C}_{-}$and $x \in D(\tilde{A}) \cap X_{1+\alpha}$. Since (3.6) is precisely (3.3) for $B=\tilde{A}$ on the space $Z$, Lemma 3.11 shows that $Z=\{0\}$.

Remark 3.12. Theorem 3.10 still holds if one replaces the assumption of polynomial stability by the hypothesis that $\sigma(A) \subset \mathbb{C}_{-}$and $\|R(\lambda, A)\| \leq \varphi(|\lambda|)$ for $\operatorname{Re} \lambda \geq 0$ and an increasing function $\varphi: \mathbb{R}_{+} \rightarrow \mathbb{R}_{+}$satisfying $\varphi(s) \leq c \exp \left(b s^{\gamma}\right)$ for constants $b, c>0$ and $0 \leq \gamma<1$. The proofs of Theorem 3.10 and Lemma 3.11 carry over to this more general setting.

Remark 3.13. Theorem 3.10, Lemma 3.11, and Remark 3.12 remain valid if we replace in the Hautus test (3.1) and in the lower estimate (3.3) the factor $m|\operatorname{Re} \lambda|$ by $\mu(|\operatorname{Re} \lambda|)$, where $\mu: \mathbb{R}_{+} \rightarrow(0, \infty)$ is an increasing unbounded function such that $\mu(r) \geq \mathrm{cr}^{\nu}$ for some constants $c, \nu>0$ and small $r>0$. One only has to observe that in the proof of Lemma 3.11 the inequality

$$
\|R(\lambda, B) z\| \leq\left[\mu\left(\frac{1}{2} \varphi(|\lambda|)^{-1}\right)\right]^{-1}\|z\|_{\alpha}
$$

holds instead of (3.4) and the inequality

$$
\|R(\lambda, B) z\| \leq \max \left\{2 \varphi(|\lambda|),\left[\mu\left(\frac{1}{2} \varphi(|\lambda|)^{-1}\right)\right]^{-1}\right\}\|z\|_{\alpha} .
$$

holds instead of (3.5). The other arguments can be used without changes. 


\section{Applications to diagonal systems}

We want to illustrate the concepts introduced in the previous section and the results established there. Let $X=\ell^{2}$ be the space of square summable complex sequences $x=\left(x_{n}\right)=\left(x_{n}\right)_{n \in \mathbb{N}}$ with the canonical basis vectors $e_{n}$. Given $\mu_{n}=-n^{-\gamma}+i n$ for $n \in \mathbb{N}=\{1,2 \cdots\}$ and some $\gamma>0$, we define $A x=\left(\mu_{n} x_{n}\right)$ for $x \in D(A)=\left\{x \in \ell^{2}\right.$ : $\left.\left(\mu_{n} x_{n}\right) \in \ell^{2}\right\}$. Then the norm $\|x\|_{\alpha}$ is equivalent to the norm $\left\|\left(n^{\alpha} x_{n}\right)\right\|_{X}$ and $A$ generates the contractive $C_{0}$-semigroup $T(\cdot)$ on $X$ given by $T(t) x=\left(e^{\mu_{n} t} x_{n}\right)$. It is easy to see that $T(\cdot)$ is polynomial stable with (sharp) constants $(\gamma, 1)$, see [3, Proposition 4.2]. Hence, (2.1) holds for $\alpha=r \gamma$ and $\beta=r$ with arbitrary $r>0$ because of (2.3). Given a complex valued sequence $\left(c_{n}\right)$, we define $C x=\sum_{n} c_{n} x_{n}$. This operator is admissible for $A$ if and only if $\left(c_{n}\right)$ is bounded, thanks to the Carleson measure criterion applied to $A-I$, see Proposition 7.1 in [22] and the references therein. We thus assume that $\left(c_{n}\right)$ is bounded.

Suppose that $\Psi_{\infty} \in \mathcal{B}\left(X_{\alpha}, L^{2}\left(\mathbb{R}_{+}\right)\right)$for some $\alpha \geq 0$. Taking $x=e_{n}$, we obtain that

$$
n^{2 \alpha} \geq \tilde{c}\left\|e_{n}\right\|_{\alpha}^{2} \geq c\left\|\Psi_{\infty} e_{n}\right\|_{L^{2}\left(\mathbb{R}_{+}\right)}^{2}=c \int_{0}^{\infty} e^{2 \operatorname{Re} \mu_{n} t}\left|c_{n}\right|^{2} d t=\frac{c n^{\gamma}}{2}\left|c_{n}\right|^{2}
$$

and thus

$$
\left|c_{n}\right| \leq \bar{c} n^{\alpha-\frac{\gamma}{2}}
$$

for some constants $c, \tilde{c}, \bar{c}>0$ and all $n \in \mathbb{N}$. (Since $c_{n}$ is bounded, condition (4.1) always holds with $\bar{c}=\left\|\left(c_{n}\right)\right\|_{\infty}$ if $\alpha \geq \gamma / 2$.) Conversely, if (4.1) is valid for all $n \in \mathbb{N}$, then the operator $C(-A)^{\gamma / 2-\alpha}$ is admissible for $A$ by the above observations. So we can estimate

$$
\begin{aligned}
\left\|\Psi_{\infty} x\right\|_{L^{2}\left(\mathbb{R}_{+}\right)}^{2} & =\sum_{k=0}^{\infty} \int_{0}^{1}\left|C(-A)^{\gamma / 2-\alpha} T(\tau) T(k)(-A)^{\alpha-\gamma / 2} x\right|^{2} d \tau \\
& \leq c \sum_{k=0}^{\infty}\left\|T(k)(-A)^{\alpha-\gamma / 2} x\right\|^{2} \\
& =c\left\|(-A)^{\alpha-\gamma / 2} x\right\|^{2}+c \sum_{k=0}^{\infty} \int_{0}^{1}\left\|T(\tau) T(k+1-\tau)(-A)^{\alpha-\gamma / 2} x\right\|^{2} d \tau \\
& \leq c\|x\|_{\alpha}^{2}+c \int_{0}^{\infty}\left\|T(t)(-A)^{\alpha-\gamma / 2} x\right\|^{2} d t \\
& =c\|x\|_{\alpha}^{2}+c \sum_{n=1}^{\infty} \int_{0}^{\infty} e^{2 \operatorname{Re} \mu_{n} t} n^{2 \alpha-\gamma}\left|x_{n}\right|^{2} d t \\
& \leq c\|x\|_{\alpha}^{2}
\end{aligned}
$$

for $x \in X_{\alpha}$ and $\alpha \geq 0$. As a result, $\Psi_{\infty}: X_{\alpha} \rightarrow L^{2}\left(\mathbb{R}_{+}\right)$is bounded if and only if (4.1) holds for all $n \in \mathbb{N}$. In particular, if $\left(c_{n}\right)$ satisfies

$$
\inf _{n \in \mathbb{N}}\left|c_{n}\right|>0 \text {, }
$$

then $\Psi_{\infty} \in \mathcal{B}\left(X_{\alpha}, L^{2}\left(\mathbb{R}_{+}\right)\right)$if and only if $\alpha \geq \gamma / 2$. So Lemma 3.1 is almost sharp.

Going back to a general admissible $C$, we assume that the system is $\alpha$-exactly infinitetime observable for some $\alpha \geq 0$. Taking $x=e_{n}$, we then deduce that

$$
n^{2 \alpha} \leq\left\|e_{n}\right\|_{\alpha}^{2} \leq c \int_{0}^{\infty}\left|e^{\mu_{n} t} c_{n}\right|^{2} d t \leq c n^{\gamma}\left|c_{n}\right|^{2}, \quad \text { hence, } \quad\left|c_{n}\right| \geq \underline{c} n^{\alpha-\frac{\gamma}{2}}
$$


for constants $c, \underline{c}>0$, obtaining a necessary condition for the $\alpha$-exact infinite-time observability of our diagonal system. In particular, $\alpha$ must be smaller or equal $\gamma / 2$ since $\left(c_{n}\right)$ is bounded. In the critical case $\alpha=\gamma / 2$, the estimate (4.3) coincides with (4.2).

An inequality analogous to (4.3) is necessary for the $(\alpha, \beta)$-Hautus test (RW). In fact, for $x=e_{n}$ and $\lambda=\mu_{n}$, this test implies that

$$
\left|c_{n}\right|^{2} \geq m^{2}\left|\operatorname{Re} \mu_{n}\right|\left|\mu_{n}\right|^{2 \beta} \geq c n^{2 \beta-\gamma}
$$

with a constant $c>0$. In particular, $\beta \leq \gamma / 2$ by the boundedness of $\left(c_{n}\right)$. Moreover, let $C$ fulfill (4.2) and the ( $\alpha, \beta)$-Hautus test (RW). Take $x=c_{n} e_{n+1}-c_{n+1} e_{n}$ and $\lambda=\mu_{n}-1$. Then $C x=0$, and so $n^{2 \beta} \leq c(n+1)^{2 \alpha}$ by this Hautus test. As a result, the $(\alpha, \beta)$-Hautus test (RW) for admissible $C$ satisfying (4.2) implies that $\alpha \geq \beta$.

We now want to apply the implication ' $(\mathrm{b}) \Rightarrow(\mathrm{a})$ ' in Proposition 3.8 to the above system. Hence we have to verify the $(\alpha, \alpha)$-Hautus test (RW) with $\alpha \in[0, \gamma / 2]$ and we have to suppose that (4.1) and (4.3) hold. In addition, we require that $\gamma>1$. Under these assumptions we will obtain the $\alpha$-exact infinite-time observability of our system for $0 \leq$ $\alpha \leq \gamma / 2$, whereas we already know that this property does not hold if $\alpha>\gamma / 2$. If we consider the borderline case $\alpha=\gamma / 2$ (where (4.2) his valid), then the above observations show that $(\alpha, \beta)$-Hautus test (RW) fails for $\alpha<\beta$ and $\beta>\gamma / 2$, in general. Moreover, Proposition 3.6 gives the optimal exponents in the Hautus test in the case $\alpha=\beta=\gamma / 2$.

In fact, we will check the $(\alpha, \alpha)$-Hautus test (RW) for $\eta C$ for some $\eta>0$ fixed below. Note that the sequence $\left(\eta c_{n}\right)$ is also bounded and satisfies (4.1) and (4.3) with $\bar{c}$ and $\underline{c}$ replaced by $\eta \bar{c}$ and $\eta \underline{c}$, respectively. So Proposition 3.8 shows the $\alpha$-exact infinite-time observability for $\eta C$ which implies the same property for $C$.

Observe that $\left|\mu_{n}-\mu_{j}\right|>1$ for all $n, j \in \mathbb{N}$ with $n \neq j$. Take $x \in X_{1+\alpha}$. First let $\operatorname{Re} \lambda \leq-2$. In this case we have

$$
\|(\lambda I-A) x\|_{\alpha}^{2}=\sum_{n=1}^{\infty}\left|\lambda-\mu_{n}\right|^{2}\left|\mu_{n}\right|^{2 \alpha}\left|x_{n}\right|^{2} \geq|\operatorname{Re} \lambda+1|^{2}\|x\|_{\alpha}^{2} \geq \frac{1}{4}|\operatorname{Re} \lambda|^{2}\|x\|_{\alpha}^{2} .
$$

Second, let $\operatorname{Re} \lambda \in(-2,0)$. Then there is at most one $n \in \mathbb{N}$ such that $\lambda \in B_{n}:=$ $B\left(\mu_{n}, \frac{1}{4}|\operatorname{Re} \lambda|\right) \subset B\left(\mu_{n}, \frac{1}{2}\right)$. If $\left|\lambda-\mu_{n}\right| \geq \frac{1}{4}|\operatorname{Re} \lambda|$ for all $n \in \mathbb{N}$, then we see as above that

$$
\|(\lambda I-A) x\|_{\alpha}^{2} \geq \frac{1}{16}|\operatorname{Re} \lambda|^{2}\|x\|_{\alpha}^{2} .
$$

It remains to consider the case that $\lambda \in B_{n}$ for some $n \in \mathbb{N}$, where $\operatorname{Re} \lambda \in(-2,0)$. Then $\left|\lambda-\mu_{j}\right| \geq 1 / 2$ for $j \neq n$. Setting $y=x-x_{n} e_{n}$, we can thus estimate

$$
\|(\lambda I-A) x\|_{\alpha}^{2} \geq \sum_{j \neq n}^{\infty}\left|\lambda-\mu_{j}\right|^{2}\left|\mu_{j}\right|^{2 \alpha}\left|x_{j}\right|^{2} \geq \frac{1}{4}\|y\|_{\alpha}^{2} .
$$

Using (4.1) and $\gamma>1$, one also deduces that $C: X_{\alpha} \rightarrow \mathbb{C}$ is bounded, say with norm $\hat{c}$. So we can further compute

$$
\begin{aligned}
|\operatorname{Re} \lambda||\eta C x|^{2} & \geq \eta^{2}|\operatorname{Re} \lambda|\left(|| c_{n} x_{n}|-| C y||\right)^{2} \geq \eta^{2}|\operatorname{Re} \lambda|\left(\left|c_{n} x_{n}\right|^{2}-2\left|c_{n} x_{n}\right||C y|\right) \\
& \geq \eta^{2}|\operatorname{Re} \lambda|\left(\frac{1}{2}\left|c_{n} x_{n}\right|^{2}-8|C y|^{2}\right) \geq \frac{1}{2} \eta^{2} \underline{c}^{2}|\operatorname{Re} \lambda|^{2}\left|n^{\alpha} x_{n}\right|^{2}-16 \eta^{2} \hat{c}^{2}\|y\|_{\alpha}^{2} .
\end{aligned}
$$

We now set $\eta=(8 \sqrt{2} \hat{c})^{-1}$, so that

$$
|\operatorname{Re} \lambda||\eta C x|^{2} \geq \frac{1}{2} \eta^{2} \underline{c}^{2}|\operatorname{Re} \lambda|^{2}\left|n^{\alpha} x_{n}\right|^{2}-\frac{1}{8}\|y\|_{\alpha}^{2} .
$$


On the other hand,

$$
m^{2}|\operatorname{Re} \lambda|^{2}\|x\|_{\alpha}^{2} \leq 2^{\alpha} m^{2}|\operatorname{Re} \lambda|^{2}\left|n^{\alpha} x_{n}\right|^{2}+4 m^{2}\|y\|_{\alpha}^{2} .
$$

Putting these estimates together and fixing a sufficiently small $m>0$, we see that the $(\alpha, \alpha)$-Hautus test (RW) holds for $\eta C$, as asserted.

Finally, we construct an approximatively infinite-time observable diagonal system which violates the $(\alpha, 0)$-Hautus test for all $\alpha \geq 0$ and every choice of $m_{\lambda}$, even in the version of Remark 3.13. Let $\alpha \geq 0$ and let $\mu$ be a function as described in Remark 3.13 with a corresponding exponent $\nu>0$. Define $A$ as above for $\mu_{n}=-\frac{2}{n}+i \frac{n}{2}$. The sequence $b_{n}=\mu(1 / n) n^{-\alpha-1 / 2}$ tends to 0 as $n \rightarrow \infty$, hence $a_{n}:=\sqrt{1-b_{n}^{2}} \in(0,1)$ for $n \geq n_{0}$ and some $n_{0} \in \mathbb{N}$. For $n \geq n_{0}$, we set $x^{n}=a_{n} e_{2 n}+b_{n} e_{2 n+1}$ so that $\left\|x^{n}\right\|=1$. For $\lambda_{n}=\mu_{2 n}$ we obtain

$$
\mu(1 / n)^{-2}\left\|\left(\lambda_{n} I-A\right) x^{n}\right\|_{\alpha}^{2}=\mu(1 / n)^{-2}\left|\mu_{2 n}-\mu_{2 n+1}\right|^{2}\left|\mu_{2 n+1}\right|^{2 \alpha} b_{n}^{2} \leq c / n .
$$

Next, define $c_{2 n}=n^{-\kappa}$ for all $n \in \mathbb{N}, c_{2 n+1}=-a_{n} c_{2 n} / b_{n}$ for $n \geq n_{0}$ and $c_{2 n+1}=1$ for $n=1, \cdots, n_{0}-1$, where $\kappa=\max \left\{1, \frac{3}{2}+\alpha+\nu\right\}$. Then $C x^{n}=0$ and the Hautus test fails for $A$ and $C$ (for arbitrary $m_{\lambda}$ ). Moreover, $\left|c_{2 n+1}\right| \leq n^{\alpha-\kappa+1 / 2} \mu(1 / n)^{-1}$ for large $n$, and so $0<\left|c_{n}\right| \leq c / n$. This fact shows that $C$ is bounded. Finally, the system given by $A$ and $C$ is approximatively infinite-time observable by [5, Thm.4.2.3].

\section{REFERENCES}

[1] F. Alabau, P. Cannarsa and V. Komornik, Indirect internal stabilization of weakly coupled evolution equations. J. Evol. Equ. 2:127-150, 2002.

[2] S.A. Avdonin and S.A. Ivanov, Families of Exponentials - The Method of Moments in Controllability Problems for Distributed Parameter Systems. Cambridge University Press, Cambridge, 1995.

[3] A. Bátkai, K.-J. Engel, J. Prüss and R. Schnaubelt, Polynomial asymptotic stability of evolution equations. Math. Nachr. (to appear).

[4] J. Conway, Functions of One Complex Variable. Second Edition, Springer-Verlag, 1978.

[5] R.F. Curtain and H.J. Zwart, An Introduction to Infinite-Dimensional Linear Systems Theory. Springer-Verlag, 1995.

[6] S. Dolecki and D.L. Russell, A general theory of observation and control. SIAM J. Control \& Optim. 15:185-220, 1977.

[7] K.-J. Engel and R. Nagel, One-Parameter Semigroups for Linear Evolution Equations. Graduate Texts in Mathematics 194, Springer-Verlag, 1999.

[8] P. Grabowski and F.M. Callier, Admissible observation operators, semigroup criteria of admissibility. Integral Equation Operator Theory 25:182-198, 1996.

[9] M.L.J. Hautus, Controllability and observability conditions for linear autonomous systems. Ned. Akad. Wetenschappen, Proc. Ser. A 72:443-448, 1969.

[10] B. Jacob and J.R. Partington, Admissibility of control and observation operators for semigroups: a survey. In J.A. Ball, J.W. Helton, M. Klaus, and L. Rodman, editors, 'Current Trends in Operator Theory and its Applications, Proceedings of IWOTA 2002,' Operator Theory: Advances and Applications 149, Birkhäuser Verlag, 199-221, 2004.

[11] B. Jacob and H. Zwart, Exact observability of diagonal systems with a finite-dimensional output operator. Systems \& Control Letters 43:101-109, 2001.

[12] B. Jacob and H. Zwart, Counterexamples concerning observation operators for $C_{0}$-semigroups. SIAM J. Control Optim. 43:137-153, 2004.

[13] B. Jacob and H. Zwart, On approximate observability of strongly-stable systems. Proc. of the MTNS 2006, Kyoto/Japan, 2006 (to appear). 
[14] V. Komornik, Exact Controllability and Stabilization - The Multiplier Method. Wiley and Masson, Chichester and Paris, 1994.

[15] Y. Latushkin, T. Randolph and R. Schnaubelt, Regularization and frequency-domain stability of well-posed control systems. Math. Control Signals Systems 17:128-151, 2005.

[16] L. Miller, Controllability cost of conservative systems: resolvent condition and transmutation. J. Funct. Anal. 218(2):425-444, 2005.

[17] A. Pazy, Semigroups of Linear Operators and Applications to Partial Differential Equation, SpringerVerlag, 1983.

[18] V.M. Popov, Hyperstability of Control Systems. Editura Academiei, Bucharest, 1966 (in Romanian), English transl. publ. by Springer-Verlag, Berlin, 1973.

[19] D.L. Russell, Controllability and stabilizability theory for linear partial differential equations: recent progress and open questions. SIAM Review 20:639-739, 1978.

[20] D.L. Russell and G. Weiss, A general necessary condition for exact observability, SIAM J. Control Optim. 32:1-23, 1994.

[21] D. Salamon, Infinite dimensional systems with unbounded control and observation: A functional analytic approach. Trans. Amer. Math. Soc. 300:388-431, 1987.

[22] G. Weiss, Admissible observation operators for linear operators. Israel J. Math. 65:17-43, 1989.

[23] Q. Zhou and M. Yamamoto, Hautus condition on the exact controllability of conservative systems. Int. J. Control 67:371-379, 1997.

Department of Applied Mathematics, Delft University of Technology, P.O. Box 5031, 2600 GA Delft, The Netherlands.

E-mail address: b.jacob@ewi.tudelft.nl

Mathematisches Institut I, Fakultät fÜr Mathematik, Universität KarlsRuhe, 76128 Karlsruhe, Germany.

E-mail address: schnaubelt@math.uni-karlsruhe.de 